Facultad de Ciencias Económicas

Universidad Nacional de La Plata

Tesis Maestría en Economía

\title{
Debilitamiento de la Clase Media: Gran Buenos Aires 1986-2004
}

\author{
Sergio Olivieri ${ }^{12}$
}

Diciembre, 2007

\begin{abstract}
Resumen
Los estudios precedentes sobre el debilitamiento de la clase media hacen uso de una definición completamente exógena para la misma. El aporte de este trabajo consiste en la implementación de la metodología desarrollada por D'Ambrosio, Muliere y Secchi (2002) que determina de manera endógena los puntos de cortes de las distintas clases sociales. Las estimaciones realizadas para el Gran Buenos Aires dan evidencia sobre el debilitamiento de la clase media entre 1992 y 2004.
\end{abstract}

\footnotetext{
${ }^{1}$ Director: Leonardo Gasparini.

${ }^{2}$ Agradezco a Walter Sosa Escudero por ayudarme a encontrar la pregunta y alentarme a investigar sobre este tema, a mi director Leonardo Gasparini por las sugerencias que mejoraron y enriquecieron el trabajo, por su paciencia y por ayudarme continuamente en el proceso, a Ana Pacheco por el aguante, largas discusiones, idas y vueltas, estados de ánimo, etc, brindándome su tiempo desinteresadamente, a Mariana Marchionni por darme ideas para mejorar el estudio, a Javier Alejo por interesarse y discutir sobre este tema generosamente, a Guillermo Cruces por empujarme a finalizar y a todo el grupo del CEDLAS por la paciencia y comprensión de estos años.
} 


\section{Introducción}

Argentina se caracterizó en el pasado por la presencia de una gran proporción de "clase media“, ubicándose entre los países con menor desigualdad en América Latina junto con Costa Rica y Uruguay. ${ }^{3}$ Minujín y Anguita (2004) establecen que esta clase social delineó una "sociedad más o menos abierta, con posibilidades de crecimiento individual y colectivo, así como de movilidad social ascendente en la que el progreso personal coronaba el esfuerzo".

En general, existe un consenso en la literatura de las ciencias sociales sobre el rol estabilizador que ejerce la "clase media". Este grupo estaría formado por aquellas personas que comparten un estatus socio-económico y pueden cooperar como también formar una voluntad política. Una clase media amplia tiene un impacto beneficioso en la sociedad debido a que actúa como un amortiguador entre las tendencias de las clases sociales baja y alta.

Durante los últimos 30 años, Argentina sufrió fuertes cambios en sus niveles de desigualdad perdiendo su lugar entre los países más igualitarios de Latinoamérica. El efecto de este empeoramiento podría interpretarse como un distanciamiento cada vez mayor entre las clases sociales del país. A su vez, esto podría generar un ambiente propicio para el desarrollo de algún tipo de conflicto o aumento de la tensión social. No existen muchos estudios con enfoque económico que traten este tema en particular para Argentina. 4

El presente trabajo atenderá principalmente a la pregunta sobre el posible debilitamiento de la clase media en el Gran Buenos Aires durante 1986 y 2004. A diferencia de los trabajos anteriores este análisis no sólo abarca un intervalo de tiempo más actual sino que utiliza una técnica de identificación de las clases sociales diferente y, en principio, menos arbitraria que sus predecesores.

Los resultados de las estimaciones mostraron que entre 1986 y 1992 la participación de la "clase media" en el Gran Buenos Aires se mantuvo relativamente constante, inclusive con una leve mejoría. Sin embargo, entre 1992 y 2004 se produjo un fuerte debilitamiento reduciendo su participación a casi la mitad.

El trabajo se organiza de la siguiente manera: en la primera sección se presenta una breve revisión de las diferentes formas en las que la literatura económica definió a la "clase media". A continuación, se describe la metodología utilizada para dividir la población del Gran Buenos Aires en 3 clases sociales: baja, media y alta. Los aspectos metodológicos y los resultados empíricos se exponen en la tercera sección del trabajo. Por último, se presentan los comentarios finales.

\footnotetext{
${ }^{3}$ Gasparini (2007)

${ }^{4}$ Referidos específicamente sobre clases sociales: Minjuín y Kessler (1995), Minujín (1995) y Minujín y Anguita (2004); Otra rama, son los estudios que analizan la polarización de la distribución del ingreso en Argentina como Horenstein y Olivieri (2004), Gertel, H., Giuliodori, R., Palacios, P. y Rulloni, V.(2006), o en Latinoamérica, Gasparini, Horenstein y Olivieri (2006) .
} 


\section{Definición de Clase Media}

El problema del debilitamiento de la "clase media" comenzó a ser estudiado por varios autores a partir de la década de los ochentas. ${ }^{5}$ La motivación de estos estudios vino dada por el fuerte incremento en los niveles de desigualdad durante un proceso de crecimiento continuo durante 7 años en Estados Unidos en dicha década. La pregunta que atienden los autores es si el proceso de crecimiento deja atrás o no a algunos sectores de la sociedad, en particular, a la "clase media".

La literatura analiza este fenómeno concentrándose en el estudio de la distribución del ingreso y salarios, principalmente, en Estados Unidos y Reino Unido. Sin embargo, la dificultad en definir la "clase media" de una sociedad originó una diversidad de metodologías para calcular su evolución en el tiempo. Estas pueden dividirse en 3 grandes grupos: aquellas que se concentran en un nivel del ingreso relativo, las que se basan sobre una proporción de la población y otras que estudian la distribución del ingreso condicionada a alguna característica respecto del ingreso medio de ese subgrupo. ${ }^{6}$

El método del primer grupo se resume en 2 pasos: primero toma como referencia al ingreso mediano y define un intervalo donde sus extremos se determinan a través de una proporción del mismo. En términos generales, los diferentes autores establecen un límite inferior de alrededor del 50\% al 75\% del ingreso mediano y un límite superior comprendido entre el $125 \%$ al $225 \%$ del ingreso mediano. En segundo lugar, miden la cantidad de personas que entran en ese intervalo y la denominan como "clase media". El descenso de esta clase se interpreta como la menor cantidad de personas que posee ingresos lo suficientemente cerca como para reflejar un estatus socio-económico similar.

El segundo enfoque determina a la clase media como el grupo de población central, por ejemplo, el 20\%, 30\% o 60\% central de la población. El deterioro se mide en términos del porcentaje de ingresos que ese grupo de la población posee. En otras palabras, dada una proporción de individuos, la clase media desaparece si su poder económico o su cohesión interna se debilitan.

El tercer grupo de estudios no define de manera precisa a la "clase media" sino que estudia la evolución de la concentración de la población condicionada a una característica, estatus laboral, nivel educativo, número de miembros del hogar, etc., en relación al ingreso medio de cada subgrupo. Este enfoque tiene, por un lado, la ventaja de evitar los problemas de definición. Sin embargo, posee la desventaja de no poder llegar a identificar cuál de todas las particiones posibles de una población es la más informativa respecto del cambio observado en la distribución del ingreso.

\footnotetext{
${ }^{5}$ Lawrence (1984), Rosenthal (1985), Blackburn y Bloom (1987), Levy (1987), Horrigan y Haugen (1988), Koster y Ross (1988), Beach (1989), Levy y Murnane (1992), Beach et.al. (1997), Jenkins (1995) y Jenkins (1996). Crews, Daly y Burkhauser (1997) realizan un estudio comparativo entre Estados Unidos y Alemania mientras que Birdsall, Graham y Pettinato (2000) para varios países del mundo. En el caso de Argentina, se encontraron algunos estudios económicos relacionados con este fenómeno: Minujín (1995), Minujín y Kessler (1995) y Minujín y Anguita (2004).

${ }^{6}$ Esta metodología se basa sobre análisis no paramétrico de las funciones de densidad del ingreso condicionadas en distintas características de la población.
} 


\section{Método de estimación de la "clase media"}

Los primeros métodos anteriormente expuestos presentan una desventaja que consiste en la definición previa por parte del investigador de lo que se entenderá por "clase social". La metodología que se utiliza en este estudio se basa sobre D' Ambrosio, Muliere y Secchi (2002) que pretende sortear este problema a través de la determinación endógena de los puntos de corte de las distintas clases sociales. Sin embargo, la desventaja que los autores advierten respecto a la implementación de esta metodología es que tanto las dimensiones de las diferentes clases sociales como sus cambios en el tiempo están sujetos al supuesto distributivo que se implemente.

En la literatura existen varios trabajos ${ }^{7}$ que requieren de un procedimiento que sea capaz de detectar distintos grupos que forman una determinada distribución. El método propuesto por D'Ambrosio, et al. (2002) establece que una serie ordenada de ingresos de la población presenta uno o varios umbrales de ingresos en los que la distribución cambia. Existen varios factores económicos y sociales que contribuyen a generar tales diferencias como, por ejemplo, el entorno social, las características tanto del hogar como las individuales, entre otras, que permitirían pensar en que individuos que pertenecen a distintas clases de ingresos provienen de diferentes poblaciones. De esta manera, por ejemplo, los ingresos de las personas pobres son generados por un mecanismo mientras que el de las personas ricas por otro.

De esta manera, sea $X=\left(X_{1}, X_{2}, \ldots, X_{n}\right)$ una serie de $n$ ingresos aleatorios de una población, $\mathrm{y} x=\left(x_{1}, x_{2}, \ldots, x_{n}\right)$ las realizaciones de $X$. Sea $\theta$ un umbral del ingreso para el cual se supone que los ingresos $X_{i} \leq \theta$ se generan por un modelo $M_{0}$ mientras que los ingresos $X_{i}>\theta$ por un modelo $M_{1}$. Según el primer modelo generador de los datos $\left(M_{0}\right)$, los ingresos $X_{i}$ son independientes e idénticamente distribuidos en forma continua con densidad $f\left(. \mid \delta_{0}\right)$. Por su parte, los ingresos generados por el segundo modelo $\left(M_{1}\right)$ también son independientes e idénticamente distribuidos en forma continua con densidad $f\left(. \mid \delta_{1}\right)$.

Para cualquier $\delta_{0} \quad$ y $\delta_{1}$ se supone que existen los números reales $0 \leq a \leq b \leq c \leq \infty$ tal que el soporte de $f\left(. \mid \delta_{0}\right) \in[a, b]$ mientras que el soporte de $f\left(. \mid \delta_{1}\right) \in(b, c)$. El umbral de ingreso $\theta$ es generalmente desconocido y debe estimarse sobre la base de las realizaciones $x$ de $X$. Con este propósito, se indica a $X_{\tau}$ al mayor ingreso entre los ingresos $X_{1}, X_{2}, \ldots, X_{n}$ que es menor o igual que $\theta$. De esta manera, $\tau$ es el punto de cambio para la serie ordenada de ingresos $X_{(1)} \leq X_{(2)} \leq \ldots \leq X_{(n)}$.

Sobre la base de las realizaciones $x$ de $X$, el punto de cambio $\tau$ se estima utilizando el método de estimación de máxima verosimilitud, siendo $\hat{\imath}(x)$ el estimador máximo verosímil y $\theta$ el umbral del ingreso con el $\hat{\imath}(x)$-ésimo nivel de ingreso entre las

\footnotetext{
7 Esteban y Ray (1994), sobre polarización, Castagnoli y Muliere (1990) y Mosler y Muliere (1996 y 1998), acerca de medidas de desigualdad consistente con versiones más débiles del principio de transferencia de Pigou-Dalton y Tsakloglou y Papadopoulos (2001), sobre exclusión social.
} 
realizaciones de $X$. Para estimar $\imath(x)$ se necesita escribir la función de máxima verosimilitud de $\tau, \delta_{0}$ y $\delta_{1}$ relativa a la serie ordenada de ingresos.

Así, sea $\left(X_{(1)}, X_{(2)}, \ldots, X_{(n)}\right)$ los estadísticos de orden de $X$, la función de densidad conjunta será:

$$
p^{\prime}\left(x_{(1)}, x_{(2)}, \ldots, x_{(n)} \mid \tau, \delta_{0}, \delta_{1}\right)=\tau ! \prod_{i=1}^{\tau} f\left(x_{(i)} \mid \delta_{0}\right) \quad(n-\tau) ! \prod_{i=\tau+1}^{n} f\left(x_{(i)} \mid \delta_{1}\right)
$$

para todo $x_{(1)} \leq x_{(2)} \leq \ldots \leq x_{(n)} \in \mathfrak{R}$. De la misma manera, se puede escribir el logaritmo de la función de máxima verosimilitud de $\tau, \delta_{0}$ y $\delta_{1}$ para las realizaciones $x=\left(x_{1}, x_{2}, \ldots, x_{n}\right)$ de $X$ :

$$
l\left(\tau, \delta_{0}, \delta_{1} \mid x_{1}, x_{2}, \ldots, x_{n}\right)=\log p^{\prime}\left(x_{(1)}, x_{(2)}, \ldots, x_{(n)} \mid \tau, \delta_{0}, \delta_{1}\right)
$$

donde $x_{(1)} \leq x_{(2)} \leq \ldots \leq x_{(n)}$ es la serie ordenada de ingresos en $x$. Equivalentemente, se puede estimar la proporción $q$ de ingresos que son generados por un modelo, por ejemplo $M_{0}$. Así, para una serie de ingresos $X=\left(X_{1}, X_{2}, \ldots, X_{n}\right)$ de tamaño $n$, el punto de cambio correspondiente a una proporción dada $q \in[0,1]$ es $\tau=[q n]$, donde $[q n]$ indica el máximo entero menor que $q n$. El logaritmo de la función de máxima verosimilitud para $q, \delta_{0}$ y $\delta_{1}$ correspondiente a las realizaciones $x$ de $X$ es:

(3) $l^{\prime}\left(q, \delta_{0}, \delta_{1} \mid x_{1}, x_{2}, \ldots, x_{n}\right)=l\left([q n], \delta_{0}, \delta_{1} \mid x_{1}, x_{2}, \ldots, x_{n}\right)=\log p^{\prime}\left(x_{(1)}, x_{(2)}, \ldots, x_{(n)} \mid[q n], \delta_{0}, \delta_{1}\right)$

donde la densidad $p^{\prime}$ y el logaritmo de la función de máxima verosimilitud $l$ están definidas por (1) y (2) respectivamente. Para poder recuperar el parámetro $\theta$ sobre la base de $x$, primero se maximiza (3) para obtener los estimadores $q(x), \delta_{0}(x)$ y $\delta_{1}(x)$. Luego, se obtiene $\hat{\imath}(x)=[\hat{q}(x) n]$ y una estimación del umbral de ingreso $\theta$ viene dado por $\hat{\theta}(x)=x_{(t(x))}$, es decir el $t(x)$-ésimo ingreso de la serie ordenada de ingresos.

D'Ambrosio, et al (2002) proponen 2 supuestos de modelos distributivos para el cálculo de los puntos de corte. El primero proviene de la combinación de 2 funciones de densidad Pareto. Así, para el primer umbral: $f\left(. \mid \delta_{0}\right)$ posee parámetros $\left(\alpha_{0}=x_{(1)}, \lambda_{0}\right)$ y está truncada en $\alpha_{1}>\alpha_{0}$ y $f\left(. \mid \delta_{1}\right)$ con parámetros $\left(\alpha_{1}=x_{([q n])}, \lambda_{1}\right)$. De esta manera, el logaritmo de la función de máxima verosimilitud (3) sería:

$$
\begin{aligned}
l^{\prime}\left(q,\left(x_{(1)}, \lambda_{0}\right),\left(x_{([q n])}, \lambda_{1}\right) \mid x_{1}, \ldots, x_{n}\right)= & =\log \left[\begin{array}{r}
\tau !\left[1-\left({ }^{x_{(1)} / x_{([q])}}\right)^{\lambda_{0}}\right]^{-\tau}\left[\prod_{i=1}^{\tau} \frac{\lambda_{0}}{x_{(1)}}\left(\frac{x_{(1)}}{x_{i}}\right)^{\lambda_{0}+1} I\left[x_{(1)}<x_{i}<x_{([q n])}\right]\right] \\
(n-\tau) !\left[\prod_{i=\tau+1}^{n} \frac{\lambda_{1}}{x_{([q n])}}\left(\frac{x_{([q n])}}{x_{i}}\right)^{\lambda_{1}+1} I\left[x_{i}>x_{([q n])}\right]\right]
\end{array}\right]
\end{aligned}
$$


El segundo modelo supone para el primer punto de corte que: $f\left(. \mid \delta_{0}\right)$ es una función de densidad Log-Normal con parámetros $\left(\mu_{0}, \sigma_{0}\right)$ y está truncada en $\alpha_{1}>0$ y $f\left(. \mid \delta_{1}\right)$ es una función Pareto con parámetros $\left(\alpha_{1}=x_{([q n])}, \lambda_{1}\right)$. Nuevamente, el logaritmo de la función de máxima verosimilitud (3) sería reemplazada por:

$$
\begin{aligned}
& l^{\prime}\left(q,\left(\mu_{0}, \sigma_{0}\right),\left(x_{([q n])}, \lambda_{1}\right) \mid x_{1}, \ldots, x_{n}\right)= \\
& =\log \left[\begin{array}{r}
\tau ! \prod_{i=1}^{\tau} \frac{1}{x_{i} \Phi\left(\sigma_{0}^{-1}\left(\log x_{([q n])}-\mu_{0}\right)\right) \sigma_{0} \sqrt{2 \pi}} \exp \left[-\frac{1}{2 \sigma_{0}^{2}}\left(\log x_{i}-\mu_{0}\right)^{2}\right] I\left[0<x_{i}<x_{([q n])}\right] \\
(n-\tau) !\left[\prod_{i=\tau+1}^{n} \frac{\lambda_{1}}{x_{([q n])}}\left(\frac{x_{([q n])}}{x_{i}}\right)^{\lambda_{1}+1} I\left[x_{i}>x_{([q n])}\right]\right]
\end{array}\right]
\end{aligned}
$$

donde $\Phi$ es la función de distribución acumulada de la normal estándar.

El método de estimación del punto de corte implementa una técnica de bootstrap. Este procedimiento se divide en 6 grandes pasos: comienza por tomar una muestra de tamaño $\mathrm{N}$ de la distribución observada de tamaño $n$ y se ordena de manera creciente; luego estima los parámetros de interés por máxima verosimilitud escogiendo un punto de corte, $\hat{q}_{1}$, en forma aleatoria y repite este proceso $\mathrm{K}$ veces, conservándose del vector de dimensión $\mathrm{K}$ sólo aquél estimador que logre el mayor valor de la función (3); se reiteran estos 2 primeros pasos $S$ veces para muestras; se obtiene la distribución del parámetro estimado, $\hat{Q}=\left(\hat{q}_{1}, \hat{q}_{2}, \ldots, \hat{q}_{S}\right)$; se estima el parámetro como la mediana de la distribución del parámetro ${ }^{8}, M e(\hat{Q})$, y este valor estimado refleja la proporción de ingresos $x=\left(x_{1}, x_{2}, \ldots, x_{n}\right)$ generados por $M_{0}$; por último, se estima el umbral $\theta$ como el $[n M e(\hat{Q})]$-ésimo ingreso de la serie ordenada de ingresos. ${ }^{9}$

\footnotetext{
${ }^{8}$ Los autores realizan 2 experimentos y observan que la mediana de la distribución del parámetro estimado se acerca mejor al verdadero valor que la media, pp 7-9

${ }_{9}^{9}$ Para más detalle y ver cómo se determinan los otros puntos de corte ver D’ Ambrosio, et al.(2002) pp. 5-6
} 


\section{Evolución de la "clase media" en Gran Buenos Aires}

\section{III.1 Aspectos Metodológicos}

Este trabajo utiliza la información proveniente de la Encuesta Permanente de Hogares (EPH) para analizar los cambios en la clase media del Gran Buenos Aires desde 1986 a 2004. Se restringió el análisis a valores positivos del ingreso adulto equivalente del hogar elaborado según el INDEC. Asimismo, se deflactaron los ingresos a pesos de 1999 utilizando el Índice de Precios al Consumidor (IPC) del Gran Buenos Aires de manera de poder hacer comparables las medidas de ingreso a través del tiempo. ${ }^{10}$

A pesar que no existen reglas generales que determinen los años que deben considerarse para realizar un análisis a lo largo del tiempo resulta importante tomar en cuenta aquellos que sean relativamente comparables. De esta manera se evitará interpretar los cambios que surgen de movimientos dentro de un ciclo económico de aquellos que ocurren entre dos momentos similares de dos ciclos consecutivos. ${ }^{11}$

Esta última tarea no parece sencilla dado que a partir de los años 1970 y durante las últimas tres décadas, Argentina experimentó, por un lado, una oscilación amplia en sus principales variables económicas ${ }^{12} \mathrm{y}$, por el otro, un fuerte deterioro en su situación socio-económica. ${ }^{13}$ No obstante y tratando de evitar problemas en la interpretación de los cambios distributivos se consideraron, por simplicidad para el análisis, sólo los años de relativa estabilidad económica separados por intervalos de tiempos equivalentes: 1986, 1992, 1998 y 2004.

Como se estableció en la sección metodológica, el análisis de las dimensiones de las clases sociales y de sus cambios está afectado directamente por el supuesto distributivo por el que se opte. Así, los supuestos funcionales adoptados para la determinación de la clase media en el Gran Buenos Aires en la mayoría de los años fueron las funciones de Pareto. En particular, para los primeros tres años de la serie

\footnotetext{
${ }^{10}$ Cuando fue necesario antes de deflactar los ingresos se convirtieron de Australes a Pesos Argentinos. Además, se consideró el promedio del IPC entre Septiembre y Octubre para aquellos años en los que la encuesta correspondía a la onda de Octubre; el promedio entre Abril y Mayo cuando la onda correspondía a Mayo; y el promedio de Julio a Diciembre cuando comprendía al segundo semestre del año.

${ }^{11}$ Daly, M., et. al (1997) y Burkhauser, R., et.al (1997)

12 Siguiendo a Gasparini (2007), luego de 2 episodios hiperinflacionarios en 1989 y 1990, la tasa de inflación cayó a menos del $1 \%$ por mes por año durante los noventa aumentando luego de la crisis del 2001. El producto bruto cayó drásticamente en el final de los 1980, creció a tasas record en el comienzo de los noventa, finalizó la década con otra recesión seguida de una fuerte crisis a finales de 2001, con una caída mayor al 15\%. Actualmente se recuperó alcanzando en 2006 niveles de actividad mayores que los de los años noventa. Por su parte, la tasa de desempleo creció persistentemente hasta 1996, tanto en un marco de crecimiento (1991-1994) como de recesión (1995-1996). Se estabilizó en un $12 \%$ al final de los noventa pero empeoró en la última crisis a niveles del $18 \%$. Actualmente, la caída en el desempleo proviene de un crecimiento sostenido en la tasa de empleo.

${ }^{13}$ Los niveles de desigualdad como los de pobreza se deterioraron durante los últimos 30 años. Si se atiende la evolución de ambos indicadores sólo para el Gran Buenos Aires, se observa que mientras la primera se mantuvo estable durante la primera mitad de los ochenta la segunda aumentó levemente. Sin embargo, ambas medidas crecieron fuertemente en la crisis hiperinflacionaria de fines de los ochenta. Luego del proceso de estabilización, la desigualdad y la pobreza cayeron. Los noventa fue otra década de aumento en la desigualdad y pobreza: el coeficiente de Gini creció 6 puntos desde 1992 a 1998 mientras que el indicador de pobreza creció 10 puntos entre 1993 y 1999. La crisis macroeconómica de 2001 volvió a generar otro aumento en otros 4 puntos y un máximo de 28 puntos para la desigualdad y pobreza respectivamente. Actualmente, por un lado, el indicador de desigualdad cayó 4 puntos en el intervalo de recuperación mientras que por el otro, el de pobreza logró alcanzar los mismos niveles de 1998. Gasparini (2007).
} 
considerada tanto el primero como el segundo punto de corte fueron estimados de manera endógena por funciones tipo Pareto. ${ }^{14}$ Sin embargo, en el caso del año 2004 se adoptó para el primer punto una combinación Log-Normal-Pareto mientras que para el segundo umbral Pareto-Pareto. ${ }^{15}$

Dado que el método de estimación implementado utiliza un procedimiento de bootstrap, es necesario establecer previamente el valor de 3 parámetros para la serie de ingresos observados $x=\left(x_{1}, x_{2}, \ldots, x_{n}\right)$ : el tamaño de la muestra aleatoria $(\mathrm{N})$, número repeticiones $(\mathrm{K})$ de la estimación de los parámetros $q, \delta_{0}$ y $\delta_{1}$ para la misma muestra aleatoria y cantidad de repeticiones de la estimación para distintas muestras (S). ${ }^{16}$ En este caso se utilizó: $\mathrm{N}=50 \%$ de la serie de ingresos observados; $\mathrm{K}=50$ repeticiones y; S $=100$ repeticiones.

\section{III.2 Resultados}

\section{Evolución y robustez}

En el Cuadro 1 se presenta la evolución de la "clase media" para el Gran Buenos Aires durante los últimos 20 años. Si se observa el cambio punta contra punta, resulta que la participación de personas que pertenecen a esta clase social se redujo un $37 \%$ a lo largo de periodo considerado. En otros términos, la "clase media" del país se debilitó y pasó de representar casi el 60\% en 1986 al 37\% en 2004. ${ }^{17}$ La misma conclusión se obtiene si se atienden a los resultados calculados sobre la base metodológica de otros trabajos pero éstos muestran una menor magnitud de decrecimiento (22\% en promedio).

El comportamiento a lo largo del periodo analizado no fue de continuo descenso. En general, éste último podría dividirse en dos etapas: la primera comprendida entre 1986 y 1992, en donde la participación de la "clase media" se mantiene relativamente constante y el resto del intervalo que muestra una fuerte caída.

Una de las ventajas de la metodología utilizada para la definición de la "clase media" es que se basa sobre la técnica de bootstrap. Esto permite armar intervalos de confianza para el umbral, mínimo y máximo, estimado y al mismo tiempo para el tamaño de la clase social analizada.

En el Cuadro 2 se muestran los puntos de corte, límites inferior y superior y las líneas de indigencia y pobreza oficiales, todos expresados en pesos de 1999. ${ }^{18} \mathrm{Si}$ se toma como referencia el umbral inferior resulta que ninguna de las estimaciones, incluidas las

\footnotetext{
${ }_{14}$ Cabe destacar se realizaron pruebas para el primer punto de corte en los años 1986-1998 sobre la base del supuesto distributivo Log Normal-Pareto, pero fueron descartadas por 2 motivos: no replicaba la heterogeneidad observada en los datos y en segundo lugar por el lento procesamiento.

${ }^{15}$ La estimación el primer umbral no pudo realizarse para una forma funcional Pareto-Pareto debido a que la solución no se encontró.

${ }^{16}$ Para más detalle sobre la metodología ver D’Ambrosio, et. al.(2002) pp. 5-6.

${ }^{17}$ Según Minujín (1995) más del 70\% de la población pertenecía a la clase media antes de la crisis de mediados de los 1970. ${ }^{18}$ Siguiendo a D' Ambrosio et al. (2002) se consideró a la mediana de la distribución del estadístico estimado como punto de corte. Además, dado que la distribución del estadístico del punto de corte no es simétrica no se utilizó el método de percentiles sino la aproximación simple que consiste en minimizar la siguiente cantidad:$$
\gamma=\frac{1}{2}\left(\hat{\theta}_{(l+0.95 B)}+\hat{\theta}_{(l+0.95 B+1)}\right)-\frac{1}{2}\left(\hat{\theta}_{(l-1)}+\hat{\theta}_{(l)}\right)
$$

respecto del entero positivo $l<0.05 B$, ver Davidson y Mackinnon (1993) pp. 763-769.
} 
de los límites inferiores, resultan ser menores a la línea de indigencia aunque si respecto de la línea de pobreza con excepción del punto estimado para el 2004.

Los puntos mínimos y máximos estimados para cada umbral permiten estimar los límites máximos y mínimos de participación de la clase media en el total de la población estudiada. En otras palabras, se estiman los intervalos de la probabilidad no condicionada de pertenecer a la clase media. Gráficamente consiste en el área debajo de la curva de densidad del ingreso equivalente comprendido entre los niveles de ingreso límites estimados (Gráfico 1).

En el Gráfico 2 se presenta la evolución de la probabilidad de pertenecer a la clase media con sus intervalos de confianza al 95\%. Así, se puede deducir que, desde un punto de vista estricto, sólo se registró un descenso significativo estadísticamente entre 1986 y 2004 debido a que no se solapan los intervalos de confianza. Sin embargo, desde un punto de vista más flexible, el proceso de debilitamiento de la "clase media" en el Gran Buenos Aires realmente acaeció entre 1992 y 2004 debido a que no existen diferencias significativas entre los niveles de 1986 y 1992. De esta manera, la clase media redujo su participación a casi la mitad pasando del 64\% al 37\% para 1992 y 2004 respectivamente.

El debilitamiento de la clase media genera un cambio en la probabilidad de pertenecer a otra clase social. En el Cuadro 3 se presentan las estimaciones de las probabilidades no condicionales de la clase baja y alta. Se observa, por un lado, que la caída en la participación de la clase media fue acompañada por un aumento de la participación de la clase baja. Por el otro lado, se registró también un descenso de la probabilidad de pertenencia de la clase alta. En resumen, entre 1992 y 2004 existió un desplazamiento de las clases superiores hacia la izquierda de la distribución del ingreso (Cuadro 3).

\section{Polarización}

En general, el análisis desarrollado hasta el momento llevaría a pensar en una sociedad más "cohesionada" debido al desplazamiento de la masa poblacional hacia la izquierda de la distribución. ${ }^{19}$ Sin embargo, si se observan los indicadores de polarización resulta que el Gran Buenos Aires registró un aumento continuo entre 1986 y 2004 (Cuadro 4).

La polarización es un concepto relacionado con el de tensión o antagonismo. Desde el punto de vista del enfoque desarrollado por Esteban y Ray (1994), comprende más de una dimensión. Una distribución se considera polarizada si posee un alto grado de homogeneidad dentro del grupo (identificación), un alto nivel de heterogeneidad entre los grupos (alienación) y un tamaño importante de los grupos. ${ }^{20}$

La razón por la que los resultados anteriores parecieran contraponerse a los de polarización, es porque hasta el momento se analizó sólo la evolución del tamaño

\footnotetext{
${ }^{19}$ Cabe notar que, en 2004, la clase baja llega a representar como máximo a tres cuartas partes de la población (Cuadro 3)

${ }^{20} \mathrm{El}$ lector interesado en las diferentes medidas de polarización y sus propiedades puede consultar Esteban y Ray (2005) y Gasparini, Horenstein y Olivieri (2006)
} 
relativo de las clases sociales olvidándose del estudio del comportamiento en las otras dos dimensiones: identificación dentro de cada clase y alienación entre las mismas.

Una manera rápida de observar estas dimensiones consiste en ver la evolución de la desigualdad dentro y entre cada clase social. Así, el primer tipo de desigualdad brinda una idea del grado de homogeneidad en las clases sociales mientras que la segunda del nivel de heterogeneidad entre los grupos.

Si se observa el Cuadro 4, resulta que la desigualdad dentro de cada clase social disminuyó levemente, entre 1992 y 2004, interpretándose como un leve aumento del grado de identificación. Por su parte, la desigualdad entre las clases sociales creció casi un $60 \%$ para el mismo periodo, traduciéndose en un ascenso en el nivel de alienación.

En grandes rasgos, por un lado el efecto de una mayor cohesión generado por el movimiento de las masas poblacionales desde las clases sociales superiores hacia la baja llevaría a una disminución del antagonismo en la sociedad. Por el otro, tanto el crecimiento leve del nivel de homogeneidad de los grupos como el aumento de la alienación intergrupal conducen a un aumento de la tensión social. En resumen, el crecimiento en los niveles de polarización vino dado porque estos últimos más que compensaron al primero.

\section{Perfiles de la Clase Media ${ }^{21}$}

La identificación de las clases sociales en grupos independientes y mutuamente excluyentes permite profundizar el análisis sobre las características que posee la "clase media", las diferencias respecto de las otras clases sociales y su variación en el tiempo. Para alcanzar este propósito se armaron perfiles para las clases sociales sobre la base de la información de la EPH de 1986 y 2004, años en los que se pudo ver un cambio estadísticamente significativo en la clase media. ${ }^{22}$

En el Cuadro 5 se presentan algunas características demográficas de la población del Gran Buenos Aires para cada clase social y ambos años. Como se vio en el apartado anterior, existió un fuerte desplazamiento de las clases superiores a la baja entre 1986 y 2004. Esto también se vio reflejado en la participación por grupos de edad resultando ser los más jóvenes quienes se vieron más afectados. Así, mientras la proporción de jóvenes menores de 15 años y de mayores de 65 de la clase media rondaba el 60 y $66 \%$ respectivamente en 1986, la participación cayó a menos del 23\% para el primer grupo y a casi el 59\% para el segundo en 2004.

La participación de la clase media pasó de ser monótona decreciente respecto de la edad en 1986 a monótona constante en 2004. Así, en el primer año el $28 \%$ de la clase media eran niños menores de 15 y el 13\% adultos mayores de 65 años en el segundo año ambos participaban con el $18 \%$. Por su parte, las otras clases sociales no variaron su

\footnotetext{
${ }^{21}$ Los perfiles que se armaron en esta sección se basan sobre los perfiles de pobreza realizados en Gasparini (2007)

22 Cabe agregar que el análisis se dirigirá principalmente a la evolución de los perfiles de la "clase media" pero cuando no se registren cambios relevantes se hará referencia al último año. Los perfiles también fueron calculados para los años 1992 y 1998 y en caso de interés pueden solicitarse.
} 
comportamiento en el intervalo de tiempo. A pesar de estos cambios, la estructura por edades sigue siendo diferente entre clases sociales lo que puede observarse a través de la edad media. Para 2004, la clase media tiene una población con 39 años de edad promedio que es un $45 \%$ superior a la de la clase baja y un $13 \%$ inferior a la clase alta.

A pesar que las clases sociales difieren en el tamaño de los hogares, esta distancia se estrecha entre las superiores. Así, en el 2004, la clase baja posee casi 2 miembros más que la clase media mientras que la clase alta no llega a tener un integrante (0.8) menos que la segunda. ${ }^{23}$ La mayor parte de la distancia se explica por la diferencia en niños menores de 12 años. En los hogares de la clase baja con jefes de edades entre 25 y 45 años existen en promedio casi 2 niños menores de 12 años mientras que en el mismo tipo de hogares de clase media no llega a haber 1 niño, en promedio y en los de clase alta sólo 0.35 en promedio. Esta diferencia en el tamaño implica que dado el mismo ingreso, una familia promedio de la clase baja con ambos padres presentes, tendría un ingreso per cápita 35\% menor al de una familia típica de clase media y un 51\% inferior al de una familia promedio de clase alta.

La tasa de dependencia ${ }^{24}$ es diferente entre clases, por ejemplo, en un hogar de clase baja cada perceptor de ingreso tenía 4 personas a su cargo en promedio mientras que tal relación caía a 2.4 y 1.8 para hogares de clase media y baja en 1986. Cabe resaltar que estas diferencias se estrecharon en el 2004.

Si se atiende a la propiedad de la vivienda en la que habita el hogar, no resultan grandes diferencias entre las clases sociales. Así, mientras el 62\% de los hogares de clase baja reportan ser propietarios, el 70\% lo hace en la clase media y el $74 \%$ en la clase alta para el 2004. En general, las personas de la clase baja habitan en viviendas con menor número de cuartos $\mathrm{y}$, como son hogares que poseen mayor número de miembros en promedio, viven en forma más hacinada que las demás.

En cuanto a la educación, los años de educación se relacionan de manera monótona creciente con la clase social a la que pertenece el individuo. Además, a lo largo de los años aumentó el nivel educativo de toda la población aunque no en la misma magnitud si se condiciona por clase social. Así, los años educativos de la clase media fueron los que más crecieron (44\%), seguidos por los de la clase alta (38\%) y clase baja (36\%). Esto llevó a que las diferencias entre los años de educación entre clases sociales aumentaran en el periodo analizado.

Estas disparidades se pueden observar más abajo del Cuadro 7. En el 2004, menos del $30 \%$ de los adultos de la clase media posee un nivel bajo de calificación, participación que se duplica si se observa a la clase baja. Por otra parte, un poco más del $75 \%$ de la población adulta de la clase alta está muy calificada frente a menos de un tercio de la clase media y sólo el $6 \%$ de la clase baja. Este último porcentaje probablemente sea menor dado que algunos profesionales al momento de realizarse la

\footnotetext{
${ }^{23} \mathrm{Si}$ se considera a 1986 como año de referencia se obtienen similares conclusiones.

${ }^{24}$ Se define como el número de miembros del hogar por perceptor de ingreso.
} 
encuesta se registran con ingresos bajos o carentes de ellos por motivos estacionales o desempleo temporal.

La tasa de alfabetismo se acerca al 100\% en todas las clases sociales para aquellas personas mayores de 10 años que reportan si saben leer y escribir, en el 2004.25 La asistencia al colegio es casi universal para los niños entre 6 y 12 años independientemente de la clase social a la que pertenezca. Sin embargo, la tasa de asistencia cae en la clase baja y media para los niveles de educación de preescolar, secundaria y terciaria respecto de los niveles alcanzados por la clase alta.

La tasa de participación en el mercado laboral crece a medida que aumenta el nivel de la clase social a la que pertenece el individuo, especialmente para las mujeres. Cabe destacar que entre 1986 y 2004, los niveles de participación de las mujeres aumentaron significativamente independientemente de la clase social considerada. La excepción en la que este indicador es menor en la clase media respecto de la baja es para los mayores de 56 años.

Además, el empleo tiene el mismo comportamiento que la tasa de participación, creciente con la clase social mientras que el desempleo una relación inversa, decreciente con la clase social. Resulta interesante observar que la tasa de desempleo total para individuos de clase baja casi triplica a la de clase media en el 2004. Estas diferencias son mayores para el caso de las mujeres. No se observan grandes diferencias en la duración del desempleo entre la clase baja y media aunque fue, en el 2004, un poco mayor para estos últimos (10.4 meses) que para los primeros (9.3 meses). Sin embargo, si se comparan con la alta (5.6 meses), la clase media tiene una duración de desempleo de alrededor del doble.

Los individuos de clase baja al conseguir trabajo lo hacen por menor cantidad de horas y menor salario horario que los de clase media y alta. En promedio, las personas de clase media al igual que las de clase alta trabajan 4.5 horas y 5.4 horas más que las de clase baja respectivamente, en 2004. Estas diferencias aumentan si se consideran a las mujeres ( 9.6 y 14.4 horas) y a individuos entre 25 y 55 años (5.1 y 7 horas) de clase media y alta. En promedio, el salario horario de una persona de clase baja es un $70 \%$ inferior a los de clase media y casi 2 veces menos que el que recibe uno de clase alta. Estas diferencias se reducen si se consideran a las mujeres y a mayores de 56 años.

Si se atiende a la estructura del empleo, resulta que los trabajadores de la clase baja son principalmente asalariados o trabajadores por cuenta propia. Los primeros se concentran en empresas pequeñas y los segundos son no calificados. Por su parte, los trabajadores de clase media presentan una composición de la relación laboral semejante a los de clase baja salvo que se distribuyen de manera distinta: los asalariados van primordialmente a empresas grandes y los por cuenta propia resultan ser más calificados que los de clase baja. Los trabajadores de clase alta se distribuyen en más de

\footnotetext{
${ }_{25}$ Cabe destacar que la EPH es una encuesta que se realiza sólo en centros urbanos del Gran Buenos Aires por lo que se esperaría una tasa más baja en el caso de incluirse las zonas rurales.
} 
tres cuartas partes entre las empresas grandes, el sector público y como profesionales independientes.

De acuerdo con la calidad del empleo que poseen los individuos, la informalidad definida sobre la base del grupo laboral al que pertenece resulta significativamente mayor para la clase baja que para las demás. Así, más del 50\% de los trabajadores de clase baja son informales mientras que tales porcentajes caen al 35\% y $16 \%$ para las clases medias y altas. En el caso en que la informalidad se defina por el acceso al seguro social, es decir que se condiciona la muestra sólo a asalariados, las diferencias a grandes rasgos aumentan dado que los de clase baja alcanzan casi el 70\% manteniéndose casi constante para el resto de las clases sociales.

Si se considera la estructura del empleo por sector económico resulta que la clase baja está distribuida entre actividades que demandan recursos humanos de baja calificación tales como la construcción, industria intensiva en mano de obra y comercio, entre otros. Por su parte, los trabajadores de clase media y alta se ubican principalmente en actividades del sector de educación y salud, comercio y servicios profesionales. 


\section{Comentarios Finales}

Este trabajo aborda el problema del debilitamiento de la "clase media" para el Gran Buenos Aires durante 1986 y 2004. Para poder responder a este interrogante el estudio se apartó del procedimiento tradicional e implementó la metodología desarrollada por D’Ambrosio, Muliere y Secchi (2002). La diferencia consiste en poder identificar de manera "endógena" los puntos de corte de las tres clases sociales en las que se dividió la distribución del ingreso para esta región de Argentina.

Los resultados de las estimaciones mostraron que, luego de la crisis de principios de siglo XXI, la estructura de la distribución del ingreso se modificó fuertemente mostrando un desplazamiento de las clases sociales superiores hacia la clase baja. Esto se tradujo en un debilitamiento a casi la mitad de la clase media del Gran Buenos Aires entre 1992 y 2004.

En términos generales, la sociedad quedó por un lado más cohesionada desde éste punto de vista: la clase baja pasó a representar casi el 58\% de la población mientras que la clase media el $37 \%$ y la clase alta un poco más del $5 \%$ restante. Por el otro lado, los niveles de polarización resultaron ser significativamente más altos que los de 1992. Esto se debió a que tanto la identificación como la alienación de las clases sociales crecieron en el periodo por lo que el antagonismo total de la sociedad terminó aumentando.

Por último, las futuras líneas de investigación comprenderían diversas aristas, como por ejemplo, proponer otras combinaciones de funciones de densidad para la estimación de los umbrales como por ejemplo, funciones betas, gama o Singh-Maddala, etc., que permitan analizar si existen cambios en las estimaciones de estos puntos de corte.

También, podría indagarse sobre las posibles causas que generaron los cambios observados en el tiempo. Los factores subyacentes de la economía que podrían causar las diferencias pueden ser varios tales como: las características de los individuos o las características de los hogares. Por lo tanto, realizar un análisis condicional de las probabilidades de pertenencia a la clase media así como también utilizar la técnica de micro-descomposición del cambio serían los pasos más apropiados para intentar dar respuesta a esta pregunta.

Además, se podría definir una medida multidimensional para identificar la "clase media". ${ }^{26}$ Se debe tener en cuenta que el nivel socio-económico que un individuo posee no está determinado sólo por su nivel de ingreso sino también por otros atributos tales como la educación, salud, empleo, condiciones de vida, etc.

${ }^{26}$ Gigliarano y Mosler (2007) proponen una metodología multivariada para la identificación de la clase media 


\section{Bibliografía}

Beach, Ch. (1989), Dollars and Dreams: A reduced middle class? Alternative Explanations, Journal of Human Resources, 24, 162-193

Beach, Ch., Chaykowski, R. y Slotsve, G. (1997), Inequality and polarization of male earnings in the United States, 1968-1990, North American Journal of Economics $\mathcal{E}$ Finance, 8, 135-151

Birdsall, N., Graham, C. y Pettinato, S. (2000) Stuck in the tunnel: Is globalization muddling the middle class?, Center on Social and Economic Dynamics, WP 14

Blackburn, M. y Bloom, D. (1987) Earnings and income inequality in the United States, Population Development Review, pp. 575-609

Burkhauser, R., Crews, A. y Daly, M. (1997) Recounting winners and losers in the 1980s: A critique of income distribution measurement methodology, Economics Letters, $54,35-40$

Burkhauser, R., Crews, A., Daly, A. y Jenkins, S. (1999) Testing the significance of income distribution changes over the 1980s business cycle: a cross-national comparison, Journal of Applied Econometrics, 14, pp 253-272

Castagnoli, E. y Muliere, P. (1990) A note on inequality measures and the Pigou-Dalton Principle of Transfers, en Dagum, C. y Zenga, M. editores, Income and Wealth Distribution, Inequality and Poverty, 171-182, Springer Verlag, Berlin

D'Ambrosio, C., Muliere, P. y Secchi, P. (2002), Income thresholds and income classes, WP, Universitá Bocconi, Milano, Italy

Daly, M., Crews, A., y Burkhauser, R. (1997) A new look at the distribution effects of economic growth during the 1980s: A comparative study of the United States and Germany, FRBSF Economic Review, Number 2, 19-31

Danzinger, S. y Gottschalk, P. (1995), America unequal, Harvard University Press, Cambridge, MA

Davidson, R. y Mackinnon, J. (1993) Estimation and inference in econometrics, Oxford University Press

Esteban, J. y Ray, D. (1994) “On the measurement of polarization” Econometrica 62, (4), Noviembre, pp. 819-852

Esteban, J. y Ray, D. (2005) A comparison of polarization measures, WP, Insitut d'Anàlisi Econòmica (CSIC), Barcelona, España

Gasparini, L. (2007) Monitoring the socio-economic conditions in Argentina 1992-2006, World Bank y CEDLAS, UNLP

Gasparini, L., Horenstein, M. y Olivieri, S. (2006) Economic Polarisation in Latin America and the Caribbean: What do Household Surveys Tell Us?, CEDLAS UNLP, La Plata, Argentina

Gasparini, L., Marchionni, M. y Sosa Escudero, W. (2005) Characterization of inequality changes through microeconometric decompositions: The case of Greater Buenos 
Aires in Bourguignon, F., Ferreira, F. y Lusting, N., The microeconomics of income distribution dynamics (In East Asia and Latin America), Oxford University Press, pp 47-83

Gertel, H., Giuliodori, R., Palacios, P. y Rulloni, V. (2006), Medidas de polarización aplicadas al estudio de la distribución del ingreso en Argentina, AAEP, Salta

Gigliarano, Ch. y Mosler, K. (2007) The declining middle class in one and many attributes, mimeo

Horenstein, M. y Olivieri, S. (2004) Income polarization in Argentina: Pure income polarization, theory and applications, Económica, Volúmen L Nro. 1-2, La Plata, Argentina

Horrigan, M. y Haugen, S. (1988), The declining middle-class thesis: a sensitivity análisis, Monthly Labor Review, 111, 3-13

Ilg, R. y Haugen, S. (2000) Earnings and employment trends in the 1990s, Monthly Labor Review, 21-33

Jenkins, S. (1995), Did the middle class shrink during the 1980s? UK evidence from kernel density estimates, Economics letters, 49, 407-413

Jenkins, S. (1996), Recent trends in the UK income distribution: what happened and why?, Oxford Review of Economic Policy, Vol. 12, No. 1

Kosters, M. y Ross, M. (1988) A shrinking middle class?, Public Interest, 90, pp 3-27

Lawrence, R. (1984) Sectoral shifts and size of the middle class, Brookings Review, pp. 3-11

Levy, F. (1987) The vanishing middle class and related issues: a review of living standards in the 1970s and 1980s, Forum, pp. 650-655

Levy, F. y Murnane, R. (1992) U.S. Earnings levels and earnings inequality: A review of recent trends and proposed explanations, Journal of Economic Literature, Vol XXX

Minujín, A. (1995), Squeezed: The middle-class in Latin America, Environment and Urbanization, vol. 7, nro 2, London

Minujín, A. y Anguita, E. (2004), La clase media: seducida y abandonada, Edhasa

Minujín, A. y Kessler, G. (1995) La nueva pobreza en Argentina, Editorial Planeta

Mosler, K. y Muliere, P. (1996) Inequality indices and the star-shaped principle transfers, Statistical Papers, 37, 343-364

Mosler, K. y Muliere, P. (1998) Welfare means and equalizing transfers, Metron, LVI N3$4,1-52$

Rosenthal, N. (1985), The shrinking middle class: myth or reality?, Monthly Labor Review, 108, 3-10

Tsakloglou, P. y Papadopoulos, F. (2001) Identifying population groups at high risk of social exclusion, IZA Discussion Paper N392 
Cuadro 1: Evolución de la proporción de personas según clase social en el Gran Buenos Aires

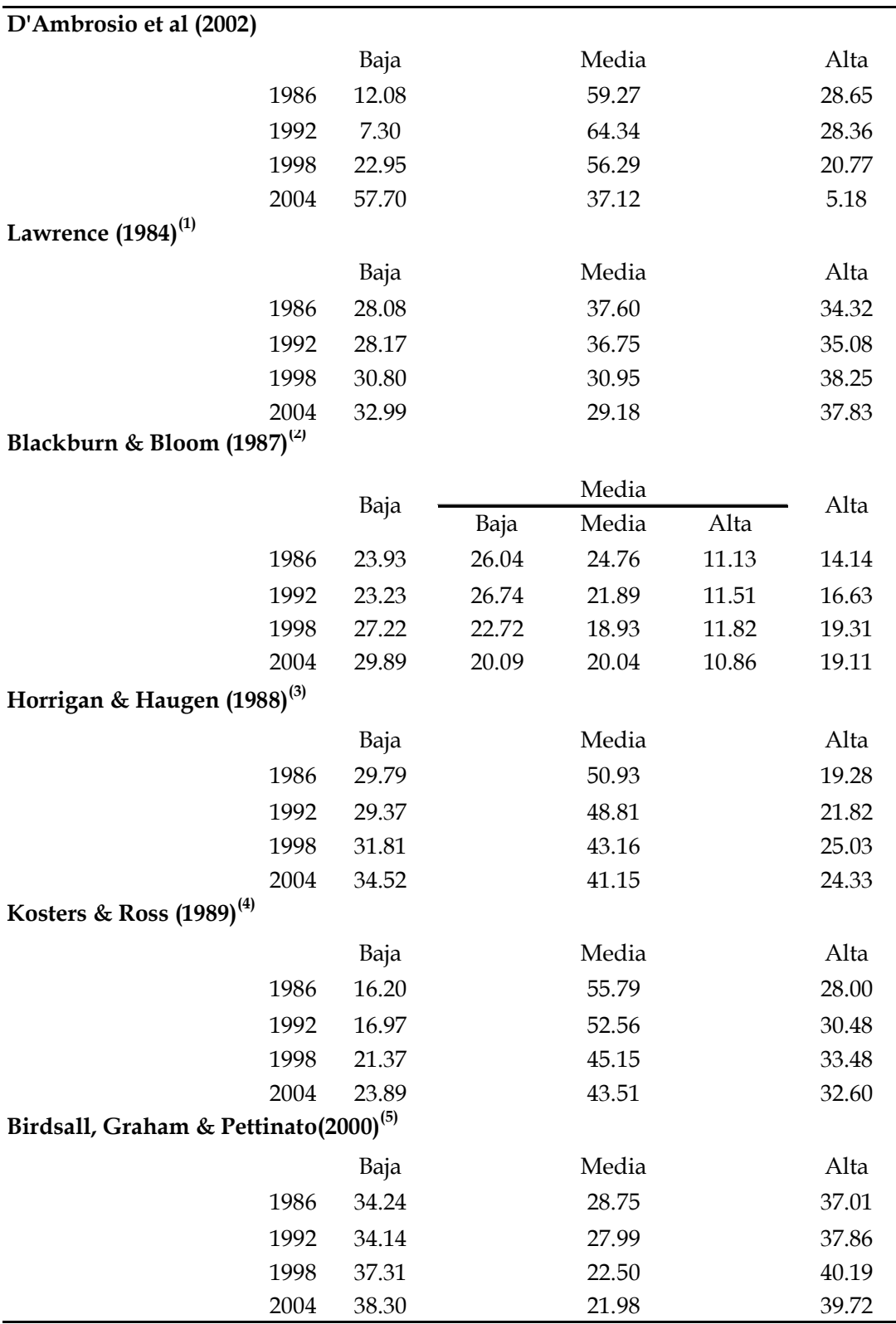

Nota: (1) Considera como límite inferior de la clase media el 66\% del ingreso mediano y el límite superior el $133 \%$ del ingreso mediano

(2) Media Baja: $60-100 \%$ de la mediana, Media: $100-160 \%$ de la mediana, Media Alta: $160-225 \%$ de la mediana

(3) Límite inferior el 68\% del ingreso mediano y límite superior el 190\% del ingreso mediano

(4) Límite inferior el $50 \%$ del ingreso mediano y límite superior el $150 \%$ del ingreso mediano

(5) Límite inferior el $75 \%$ del ingreso mediano y límite superior el $125 \%$ del ingreso mediano Fuente: Elaboración propia sobre la base de EPH 
Cuadro 2: Puntos de corte y métodos de estimación - en pesos de 1999

\begin{tabular}{|c|c|c|c|c|c|c|c|c|c|c|}
\hline & \multirow{2}{*}{ Minimo } & \multicolumn{2}{|c|}{ Intervalo 95\% } & \multirow{2}{*}{ Maximo } & \multicolumn{2}{|c|}{ Intervalo 95\% } & \multicolumn{2}{|c|}{ Lineas } & \multicolumn{2}{|c|}{ Método } \\
\hline & & $\min$ & $\max$ & & $\min$ & $\max$ & Indigencia & Pobreza & 1 punto & 2 punto \\
\hline 1986 & 174.33 & 147.74 & 188.17 & 571.05 & 538.69 & 653.75 & 76.84 & 177.50 & $\mathrm{PP}$ & $\mathrm{PP}$ \\
\hline 1992 & 109.77 & 103.15 & 117.54 & 471.08 & 349.86 & 615.08 & 57.94 & 129.21 & PP & PP \\
\hline 1998 & 146.48 & 86.26 & 167.61 & 598.05 & 540.70 & 902.46 & 69.78 & 161.19 & PP & PP \\
\hline 2004 & 240.85 & 202.73 & 379.48 & 934.52 & 639.86 & $1,233.40$ & 108.54 & 237.70 & LP & PP \\
\hline
\end{tabular}

Nota: $\mathrm{PP}=$ Pareto-Pareto

LP=LogNormal-Pareto

Fuente: Elaboración propia sobre la base de EPH

Cuadro 3: Intervalo de mínima y máxima participación de cada clase social

\begin{tabular}{|c|c|c|c|c|c|c|c|c|c|}
\hline & \multirow{2}{*}{$\begin{array}{c}\text { Clase Baja } \\
P(y<m)\end{array}$} & \multicolumn{2}{|c|}{ Intervalo } & \multirow{2}{*}{$\begin{array}{l}\text { Clase Media } \\
P(m<y<M)\end{array}$} & \multicolumn{2}{|c|}{$\begin{array}{l}\text { Intervalo } \\
\end{array}$} & \multirow{2}{*}{$\begin{array}{l}\text { Clase Alta } \\
P(y>M)\end{array}$} & \multicolumn{2}{|c|}{ Intervalo } \\
\hline & & $P\left(y<m_{(\min )}\right)$ & $P\left(y<m_{(\max )}\right)$ & & $P\left(m_{(\max )}<y<M_{(\min )}\right)$ & $P\left(m_{(\min )}<y<M_{(\max )}\right)$ & & $P\left(y>M_{(\max )}\right)$ & $P\left(y>M_{(\min )}\right)$ \\
\hline 1986 & 12.1 & 8.6 & 15.4 & 59.3 & 52.8 & 68.6 & 28.7 & 22.8 & 31.8 \\
\hline 1992 & 7.3 & 6.2 & 9.6 & 64.3 & 48.9 & 74.5 & 28.4 & 19.3 & 41.6 \\
\hline 1998 & 22.9 & 9.7 & 27.6 & 56.3 & 48.3 & 79.6 & 20.8 & 10.7 & 24.2 \\
\hline 2004 & 57.7 & 49.7 & 75.0 & 37.1 & 13.7 & 47.7 & 5.2 & 2.6 & 11.3 \\
\hline
\end{tabular}

Fuente: Elaboración propia sobre la base de EPH

Cuadro 4: Evolución de la desigualdad y polarización del ingreso en el Gran Buenos Aires

\begin{tabular}{|c|c|c|c|c|c|c|c|c|}
\hline & \multirow{2}{*}{ Gini } & \multicolumn{3}{|c|}{ Theil } & \multirow{2}{*}{$\mathrm{CV}$} & \multicolumn{3}{|c|}{ Atkinson } \\
\hline & & Intra (wth) & Inter (btw) & Total & & 0.50 & 1 & 2 \\
\hline 1986 & 0.414 & 0.104 & 0.209 & 0.312 & 0.985 & 0.140 & 0.254 & 0.432 \\
\hline 1992 & 0.443 & 0.127 & 0.239 & 0.365 & 1.138 & 0.160 & 0.287 & 0.486 \\
\hline 1998 & 0.502 & 0.133 & 0.340 & 0.473 & 1.303 & 0.207 & 0.367 & 0.605 \\
\hline \multirow[t]{3}{*}{2004} & 0.507 & 0.120 & 0.380 & 0.500 & 1.558 & 0.214 & 0.378 & 0.621 \\
\hline & \multirow{2}{*}{ Wolfson } & \multirow{2}{*}{ ZK } & \multirow{2}{*}{ EGR2 } & \multirow{2}{*}{ EGR3 } & \multicolumn{4}{|c|}{ DER } \\
\hline & & & & & 0.25 & 0.50 & 0.75 & 1 \\
\hline 1986 & 0.363 & 2.014 & 0.132 & 0.513 & 0.305 & 0.249 & 0.215 & 0.193 \\
\hline 1992 & 0.405 & 1.882 & 0.151 & 0.554 & 0.324 & 0.265 & 0.232 & 0.213 \\
\hline 1998 & 0.493 & 2.561 & 0.173 & 0.630 & 0.358 & 0.289 & 0.251 & 0.229 \\
\hline 2004 & 0.488 & 3.169 & 0.173 & 0.636 & 0.360 & 0.290 & 0.251 & 0.226 \\
\hline
\end{tabular}

Fuente: Elaboración propia sobre la base de EPH 
Cuadro 5: Perfiles de clases sociales. Demografía

\begin{tabular}{|c|c|c|c|c|c|c|}
\hline & \multicolumn{3}{|c|}{1986} & \multicolumn{3}{|c|}{2004} \\
\hline & Baja & Media & Alta & Baja & Media & Alta \\
\hline Porcentaje de población & 12.08 & 59.27 & 28.65 & 57.73 & 37.09 & 5.18 \\
\hline \multicolumn{7}{|c|}{ Porcentaje de población por edad } \\
\hline$[0,15]$ & 20.44 & 60.00 & 19.56 & 75.22 & 22.83 & 1.95 \\
\hline$[16,25]$ & 11.45 & 61.81 & 26.74 & 62.58 & 34.26 & 3.16 \\
\hline$[26,40]$ & 11.54 & 54.91 & 33.55 & 54.44 & 39.38 & 6.18 \\
\hline$[41,64]$ & 7.55 & 57.68 & 34.77 & 48.49 & 44.06 & 7.45 \\
\hline$[65+]$ & 3.85 & 66.07 & 30.09 & 31.47 & 58.78 & 9.75 \\
\hline \multicolumn{7}{|l|}{ Distribución por edad } \\
\hline$[0,15]$ & 46.79 & 27.99 & 18.88 & 37.28 & 17.61 & 10.78 \\
\hline$[16,25]$ & 12.90 & 14.19 & 12.70 & 17.40 & 14.82 & 9.78 \\
\hline$[26,40]$ & 20.85 & 20.21 & 25.54 & 20.00 & 22.51 & 25.31 \\
\hline$[41,64]$ & 15.71 & 24.46 & 30.49 & 19.13 & 27.06 & 32.74 \\
\hline$[65+]$ & 3.75 & 13.14 & 12.38 & 6.19 & 18.00 & 21.38 \\
\hline Total & 100.00 & 100.00 & 100.00 & 100.00 & 100.00 & 100.00 \\
\hline Edad Promedio & 23.22 & 33.68 & 37.07 & 27.10 & 39.34 & 44.27 \\
\hline \multicolumn{7}{|c|}{ Tamaño de hogar y estructura } \\
\hline Tamaño de la familia & 5.64 & 4.25 & 3.42 & 5.06 & 3.26 & 2.49 \\
\hline Niños menores de 12 años & 2.68 & 1.63 & 1.01 & 1.81 & 0.85 & 0.35 \\
\hline Tasa de dependencia & 4.14 & 2.38 & 1.76 & 2.88 & 1.62 & 1.30 \\
\hline
\end{tabular}

Cuadro 6: Perfiles de clases sociales. Vivienda

\begin{tabular}{|c|c|c|c|c|c|c|}
\hline & \multicolumn{3}{|c|}{1986} & \multicolumn{3}{|c|}{2004} \\
\hline & Baja & Media & Alta & Baja & Media & Alta \\
\hline Propietario & 61.09 & 72.74 & 74.99 & 62.72 & 69.45 & 73.60 \\
\hline Número de habitaciones & 2.51 & 2.71 & 3.14 & 2.69 & 2.90 & 3.24 \\
\hline Personas por cuarto & 2.23 & 1.39 & 0.93 & 1.80 & 0.97 & 0.62 \\
\hline
\end{tabular}

Fuente: Elaboración propia sobre la base de EPH 
Cuadro 7: Perfiles de clases sociales. Educación

\begin{tabular}{|c|c|c|c|c|c|c|}
\hline & \multicolumn{3}{|c|}{1986} & \multicolumn{3}{|c|}{2004} \\
\hline & Baja & Media & Alta & Baja & Media & Alta \\
\hline \multicolumn{7}{|l|}{ Años de educación } \\
\hline Total & 4.67 & 6.43 & 9.30 & 6.35 & 9.25 & 12.83 \\
\hline$[10,20]$ & 6.46 & 7.42 & 8.16 & 7.45 & 8.48 & 9.20 \\
\hline$[21,30]$ & 7.12 & 9.44 & 12.45 & 9.57 & 12.38 & 15.04 \\
\hline$[31,40]$ & 6.86 & 8.88 & 12.80 & 8.86 & 12.46 & 15.64 \\
\hline$[41,50]$ & 6.19 & 7.66 & 11.29 & 8.37 & 11.31 & 15.21 \\
\hline$[51,60]$ & 6.00 & 6.72 & 9.35 & 6.87 & 10.17 & 14.71 \\
\hline$[61+]$ & 5.04 & 6.24 & 8.32 & 6.17 & 7.97 & 12.23 \\
\hline \multicolumn{7}{|l|}{ Educación por grupos } \\
\hline \multicolumn{7}{|l|}{ Adultos } \\
\hline Bajo & 79.58 & 64.45 & 30.85 & 59.73 & 27.86 & 4.98 \\
\hline Medio & 19.10 & 29.55 & 38.78 & 33.93 & 40.29 & 19.46 \\
\hline Alto & 1.32 & 6.00 & 30.37 & 6.33 & 31.86 & 75.56 \\
\hline Total & 100.00 & 100.00 & 100.00 & 100.00 & 100.00 & 100.00 \\
\hline \multicolumn{7}{|l|}{ Hombres adultos } \\
\hline Bajo & 79.75 & 59.97 & 27.81 & 61.05 & 28.18 & 6.13 \\
\hline Medio & 19.11 & 32.61 & 36.10 & 33.55 & 43.48 & 19.88 \\
\hline Alto & 1.14 & 7.42 & 36.09 & 5.40 & 28.34 & 73.98 \\
\hline Total & 100.00 & 100.00 & 100.00 & 100.00 & 100.00 & 100.00 \\
\hline \multicolumn{7}{|l|}{ Mujeres adultas } \\
\hline Bajo & 79.43 & 68.38 & 33.49 & 58.55 & 27.57 & 3.94 \\
\hline Medio & 19.08 & 26.86 & 41.10 & 34.28 & 37.49 & 19.07 \\
\hline Alto & 1.49 & 4.76 & 25.41 & 7.17 & 34.94 & 76.99 \\
\hline Total & 100.00 & 100.00 & 100.00 & 100.00 & 100.00 & 100.00 \\
\hline \multicolumn{7}{|l|}{ Jefes de Hogar } \\
\hline Bajo & 80.52 & 66.94 & 35.41 & 63.99 & 40.01 & 8.92 \\
\hline Medio & 18.51 & 27.45 & 35.74 & 31.61 & 36.76 & 26.74 \\
\hline Alto & 0.98 & 5.60 & 28.85 & 4.40 & 23.24 & 64.34 \\
\hline Total & 100.00 & 100.00 & 100.00 & 100.00 & 100.00 & 100.00 \\
\hline Tasa de alfabetismo & 95.58 & 97.59 & 99.55 & 98.27 & 99.27 & 99.82 \\
\hline \multicolumn{7}{|l|}{ Asistencia } \\
\hline$[3,5]$ & 14.60 & 19.38 & 29.57 & 62.52 & 81.24 & 100.00 \\
\hline$[6,12]$ & 93.64 & 97.08 & 96.35 & 98.41 & 100.00 & 100.00 \\
\hline$[13,17]$ & 68.81 & 74.48 & 89.79 & 87.76 & 94.90 & 100.00 \\
\hline$[18,23]$ & 26.85 & 30.58 & 47.56 & 24.91 & 49.22 & 76.30 \\
\hline
\end{tabular}

Fuente: Elaboración propia sobre la base de EPH 
Cuadro 8: Perfiles de clases sociales. Empleo

\begin{tabular}{|c|c|c|c|c|c|c|}
\hline & \multicolumn{3}{|c|}{1986} & \multicolumn{3}{|c|}{2004} \\
\hline & Baja & Media & Alta & Baja & Media & Alta \\
\hline \multicolumn{7}{|l|}{ Fuerza Laboral } \\
\hline Total & 24.86 & 34.58 & 51.06 & 53.39 & 60.05 & 69.87 \\
\hline$[16,24]$ & 39.73 & 51.59 & 57.72 & 56.05 & 62.86 & 57.16 \\
\hline$[25,55]$ & 56.49 & 67.33 & 80.66 & 77.77 & 86.10 & 96.13 \\
\hline$[56+]$ & 23.68 & 16.51 & 36.06 & 36.12 & 32.13 & 42.25 \\
\hline Hombres $[25,55]$ & 91.34 & 97.32 & 97.84 & 95.92 & 96.74 & 99.29 \\
\hline Mujeres [25,55] & 24.63 & 39.71 & 65.85 & 61.54 & 76.33 & 93.31 \\
\hline \multicolumn{7}{|l|}{ Empleados } \\
\hline Total & 20.59 & 33.02 & 50.33 & 42.41 & 55.52 & 69.28 \\
\hline$[16,24]$ & 27.53 & 47.64 & 55.96 & 34.20 & 51.80 & 55.53 \\
\hline$[25,55]$ & 49.18 & 65.28 & 79.75 & 65.87 & 81.61 & 95.56 \\
\hline$[56+]$ & 17.63 & 15.57 & 35.56 & 30.22 & 29.64 & 41.82 \\
\hline Hombres $[25,55]$ & 82.43 & 94.92 & 96.66 & 85.04 & 93.49 & 98.72 \\
\hline Mujeres $[25,55]$ & 18.79 & 37.96 & 65.18 & 48.71 & 70.70 & 92.75 \\
\hline \multicolumn{7}{|l|}{ Tasa de desempleo } \\
\hline Total & 17.15 & 4.53 & 1.42 & 20.56 & 7.54 & 0.84 \\
\hline$[16,24]$ & 30.72 & 7.65 & 3.05 & 38.98 & 17.59 & 2.85 \\
\hline$[25,55]$ & 12.94 & 3.06 & 1.13 & 15.31 & 5.22 & 0.59 \\
\hline$[56+]$ & 25.56 & 5.72 & 1.38 & 16.32 & 7.76 & 1.01 \\
\hline Hombres $[25,55]$ & 9.76 & 2.46 & 1.20 & 11.34 & 3.36 & 0.57 \\
\hline Mujeres $[25,55]$ & 23.70 & 4.39 & 1.03 & 20.84 & 7.38 & 0.60 \\
\hline $\begin{array}{l}\text { Duración del desempleo } \\
\text { (en meses) }\end{array}$ & 1.99 & 1.80 & 1.77 & 9.28 & 10.44 & 5.60 \\
\hline
\end{tabular}

Fuente: Elaboración propia sobre la base de EPH

Cuadro 9: Perfiles de clases sociales. Horas, salarios horarios e ingreso laboral

\begin{tabular}{|c|c|c|c|c|c|c|}
\hline & \multicolumn{3}{|c|}{1986} & \multicolumn{3}{|c|}{2004} \\
\hline & Baja & Media & Alta & Baja & Media & Alta \\
\hline \multicolumn{7}{|l|}{ Horas trabajadas } \\
\hline Total & 44.58 & 46.24 & 45.08 & 37.97 & 42.45 & 43.38 \\
\hline$[16,24]$ & 42.76 & 43.03 & 39.95 & 37.60 & 39.69 & 30.10 \\
\hline$[25,55]$ & 46.08 & 47.69 & 46.29 & 38.87 & 43.98 & 45.89 \\
\hline$[56+]$ & 38.55 & 42.18 & 43.93 & 33.87 & 38.65 & 39.26 \\
\hline Hombres $[25,55]$ & 49.42 & 51.49 & 51.42 & 45.74 & 49.09 & 49.52 \\
\hline Mujeres [25,55] & 32.28 & 38.72 & 39.60 & 28.04 & 37.65 & 42.46 \\
\hline \multicolumn{7}{|c|}{ Salario horario (en pesos de 1999) } \\
\hline Total & 2.33 & 3.64 & 7.68 & 2.06 & 3.51 & 9.25 \\
\hline$[16,24]$ & 2.00 & 2.74 & 4.50 & 1.60 & 2.45 & 4.53 \\
\hline$[25,55]$ & 2.47 & 3.95 & 8.01 & 2.13 & 3.75 & 8.02 \\
\hline$[56+]$ & 1.54 & 3.35 & 8.80 & 2.39 & 3.37 & 14.67 \\
\hline Hombres $[25,55]$ & 2.38 & 4.14 & 9.40 & 2.12 & 3.93 & 9.07 \\
\hline Mujeres $[25,55]$ & 2.84 & 3.50 & 6.24 & 2.14 & 3.54 & 7.01 \\
\hline \multicolumn{7}{|c|}{ Ingresos Laborales (en pesos de 1999) } \\
\hline Total & 384.85 & 665.68 & $1,277.60$ & 264.35 & 557.68 & $1,457.24$ \\
\hline$[16,24]$ & 293.29 & 466.49 & 696.81 & 195.12 & 343.63 & 507.62 \\
\hline$[25,55]$ & 426.30 & 741.92 & $1,448.94$ & 289.23 & 623.11 & $1,458.48$ \\
\hline$[56+]$ & 218.65 & 525.00 & $1,044.03$ & 216.99 & 459.53 & $1,733.49$ \\
\hline Hombres $[25,55]$ & 467.43 & 850.34 & $1,851.03$ & 336.33 & 731.88 & $1,757.95$ \\
\hline Mujeres $[25,55]$ & 253.39 & 491.71 & 950.16 & 194.40 & 487.99 & $1,169.09$ \\
\hline
\end{tabular}

Fuente: Elaboración propia sobre la base de EPH 
Cuadro 10: Perfiles de clases sociales. Estructura del empleo

\begin{tabular}{|c|c|c|c|c|c|c|}
\hline & \multicolumn{3}{|c|}{1986} & \multicolumn{3}{|c|}{2004} \\
\hline & Baja & Media & Alta & Baja & Media & Alta \\
\hline \multicolumn{7}{|l|}{ Relación laboral } \\
\hline Patrón & 0.27 & 2.15 & 8.18 & 0.91 & 3.12 & 7.70 \\
\hline Asalariado & 59.10 & 71.41 & 69.22 & 61.02 & 73.54 & 72.69 \\
\hline Cuenta propia & 23.21 & 20.61 & 19.96 & 16.44 & 15.34 & 18.77 \\
\hline Sin ingreso & 0.27 & 1.28 & 1.21 & 1.06 & 0.45 & - \\
\hline Desempleado & 17.15 & 4.54 & 1.43 & 20.56 & 7.54 & 0.84 \\
\hline Total & 100.00 & 100.00 & 100.00 & 100.00 & 100.00 & 100.00 \\
\hline \multicolumn{7}{|l|}{ Por grupo laboral } \\
\hline Patrón & 0.35 & 2.43 & 8.85 & 1.21 & 3.50 & 7.92 \\
\hline Asalariado-empresa grande & 46.35 & 51.71 & 55.36 & 28.33 & 40.73 & 45.22 \\
\hline Asalariado-sector público & - & - & - & 17.77 & 16.50 & 19.86 \\
\hline Cuenta propia-profesionales & - & 0.59 & 4.36 & 0.63 & 4.06 & 11.23 \\
\hline Asalariado-empresa chica & 22.97 & 21.18 & 12.88 & 29.57 & 21.55 & 7.70 \\
\hline Cuenta propia-sin calificación & 29.98 & 22.65 & 17.24 & 21.09 & 13.14 & 8.07 \\
\hline Sin ingreso & 0.35 & 1.45 & 1.31 & 1.41 & 0.50 & - \\
\hline Total & 100.00 & 100.00 & 100.00 & 100.00 & 100.00 & 100.00 \\
\hline \multicolumn{7}{|c|}{ Formalidad (sobre la base del grupo laboral) } \\
\hline Formal & 46.70 & 54.72 & 68.57 & 47.93 & 64.80 & 84.23 \\
\hline Informal & 53.30 & 45.28 & 31.43 & 52.07 & 35.20 & 15.77 \\
\hline Total & 100.00 & 100.00 & 100.00 & 100.00 & 100.00 & 100.00 \\
\hline \multicolumn{7}{|c|}{ Formalidad (sobre la base derecho a jubilación) } \\
\hline Formal & 69.47 & 73.06 & 84.88 & 31.76 & 64.90 & 77.85 \\
\hline Informal & 30.53 & 26.94 & 15.12 & 68.24 & 35.10 & 22.15 \\
\hline Total & 100.00 & 100.00 & 100.00 & 100.00 & 100.00 & 100.00 \\
\hline \multicolumn{7}{|l|}{ Sectores } \\
\hline Actividades primarias & 0.82 & 0.24 & 0.38 & 1.00 & 0.28 & 1.65 \\
\hline Industria-intensiva en trabajo & 16.24 & 12.29 & 8.30 & 11.28 & 8.24 & 2.78 \\
\hline Industria-intensiva en capital & 17.39 & 16.45 & 16.63 & 7.65 & 8.71 & 7.47 \\
\hline Construcción & 16.19 & 8.15 & 3.65 & 11.20 & 4.07 & 1.41 \\
\hline Comercio & 20.48 & 21.45 & 20.44 & 26.32 & 21.80 & 12.83 \\
\hline Transporte y comunicaciones & 5.24 & 6.83 & 5.10 & 6.96 & 8.45 & 7.43 \\
\hline Servicios profesionales & 1.73 & 5.51 & 15.25 & 4.08 & 11.20 & 22.79 \\
\hline Administración Pública & 1.67 & 4.14 & 5.07 & 6.35 & 7.13 & 11.66 \\
\hline Educación \& Salud & 9.11 & 14.13 & 21.01 & 15.19 & 22.64 & 31.53 \\
\hline Servicio doméstico & 11.13 & 10.82 & 4.18 & 9.98 & 7.48 & 0.47 \\
\hline Total & 100.00 & 100.00 & 100.00 & 100.00 & 100.00 & 100.00 \\
\hline
\end{tabular}


Gráfico 1: Probabilidad de pertenecer a la clase media en el Gran Buenos Aires Año 1986 - intervalo del 95\%
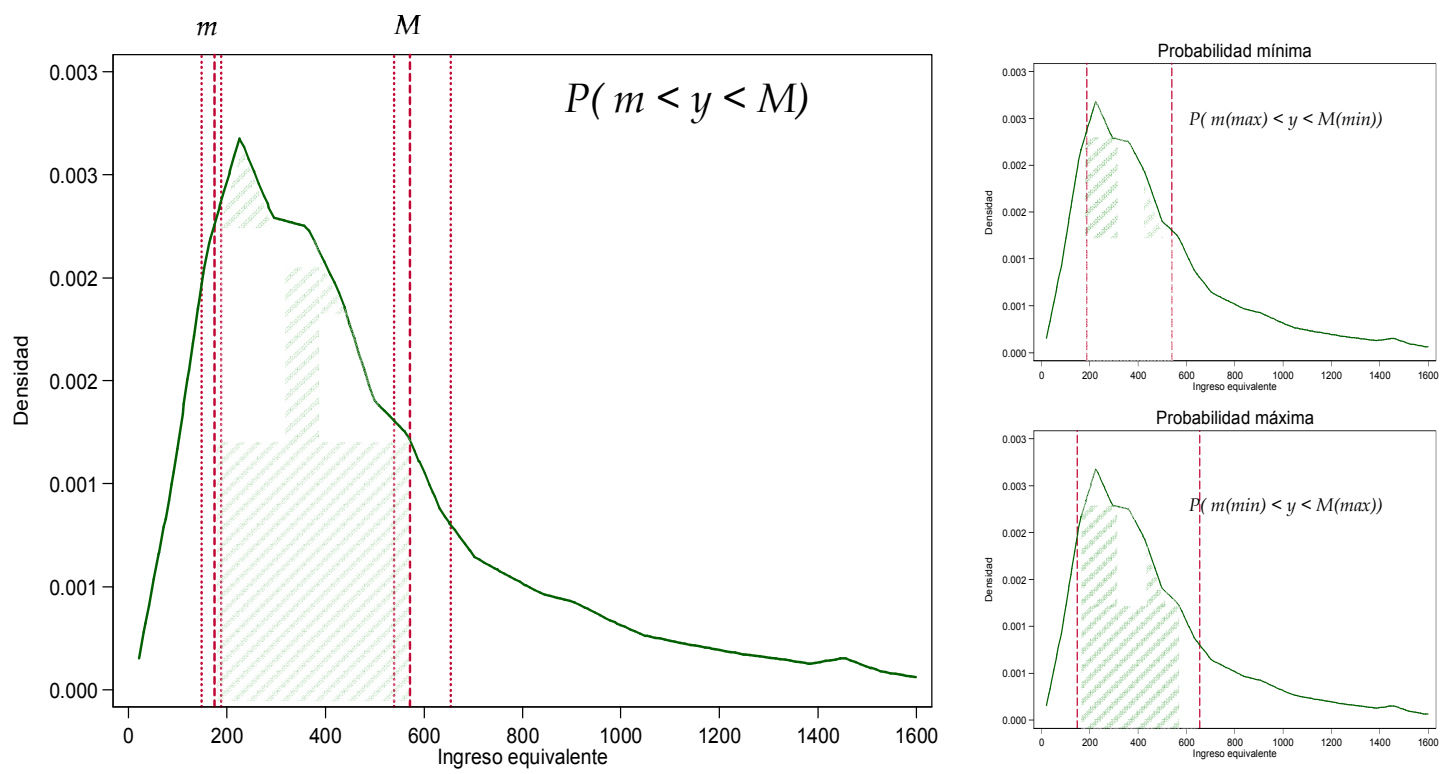

Fuente: Elaboración propia sobre la base de EPH

Gráfico 2: Evolución de la proporción de personas clase media en el Gran Buenos Aires - intervalo del 95\%

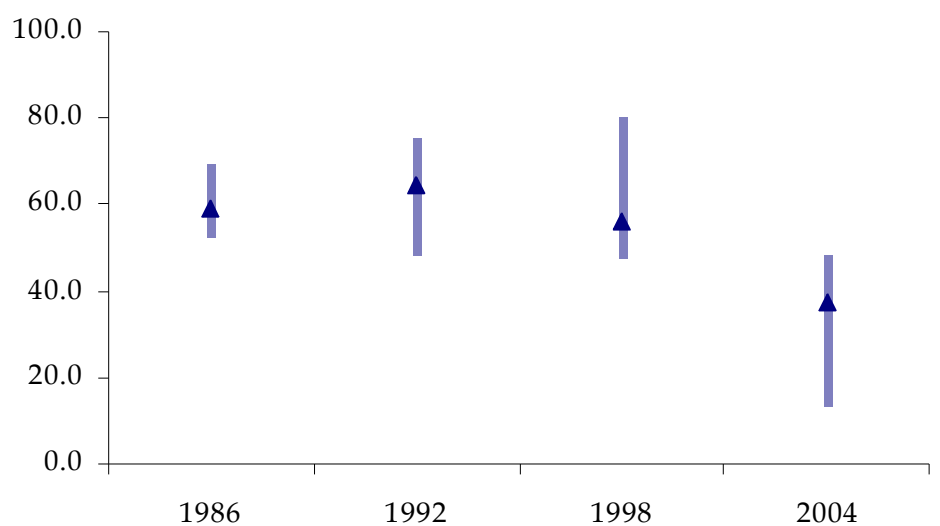

Fuente: Elaboración propia sobre la base de EPH 